\title{
Reactive oxygen species regulate Bax translocation and mitochondrial transmembrane potential, a possible mechanism for enhanced TRAIL-induced apoptosis by CCCP
}

\author{
ATUL A. CHAUDHARI, JAE-WON SEOL, SHANG-JIN KIM, YOU-JIN LEE, \\ HYUNG-SUB KANG, IN-SHIK KIM, NAM-SOO KIM and SANG-YOUEL PARK \\ Center for Healthcare Technology Development, Bio-Safty Research Institute College of \\ Veterinary Medicine, Chonbuk National University, Jeonju, Jeonbuk 561-756, South Korea
}

Received January 12, 2007; Accepted April 25, 2007

\begin{abstract}
TRAIL is a TNF family member that engages apoptosis via recruitment and rapid activation of caspase-8. Oxygen-free radicals, more generally known as reactive oxygen species (ROS) are well recognized for playing an important role in the regulation of tumor cell apoptosis. ROS within the cells act as secondary messengers in intracellular signalling cascades therefore function as anti-tumorigenic species. But very little is known about the effect of ROS on TRAIL-induced apoptosis. In this study we investigated the effect of CCCP, a classic uncoupler of oxidative phosphorylation, on TRAIL-induced apoptosis in TRAIL-resistant MCF-7 cells. It was found that pretreatment with CCCP for $6 \mathrm{~h}$ and then treatment with TRAIL for additional $3 \mathrm{~h}$ markedly enhanced apoptosis by 2 -fold as compared with TRAIL alone. The uncoupling effect enhanced TRAIL-induced apoptosis by ROS generation. Moreover, CCCP treatment also reduced mitochondrial transmembrane potential (MTP, $\Delta \Psi \mathrm{m})$ and induced Bax translocation to the mitochondria of its own account. This sensitization was inhibited with $\mathrm{N}$-acetyl-L-cysteine (NAC) treatment by abrogating the ROS which was generated by the combined treatment of CCCP and TRAIL. Of interest, NAC also inhibited reduction of the $\triangle \Psi \mathrm{m}$ and Bax translocation after CCCP pretreatment which suggest that the generartion of ROS may precede the loss in MTP. Thus, we demonstrated that CCCP-induced ROS generation enhanced TRAIL induced apoptosis by regulation of Bax translocation and mitochondrial transmembrane potential. The enhancing effect by CCCP-induced ROS generation was restored after NAC treatment. Therefore, our results suggest that uncoupling the cells by CCCP can overcome the resistance to TRAIL protein and can be a very efficient treatment for the tumor cells especially resistant to TRAIL-induced apoptosis.
\end{abstract}

Correspondence to: Dr Sang-Youel Park, College of Veterinary Medicine, Chonbuk National University, Jeonju, Jeonbuk 561-756, South Korea

E-mail:sypark@chonbuk.ac.kr

Key words: ROS, TRAIL, apoptosis, mitochondrial transmembrane potential, Bax

\section{Introduction}

Apoptosis is a tightly regulated cell death program that plays a pivotal role in a variety of biological processes. Unlike necrosis, apoptosis is a fundamental biochemical process involving the selective and controlled elimination of cells in multicellular organisms during normal cellular differentiation and development (1-3). Apoptosis is characterised by membrane blebbing, nuclear fragmentation, formation of apoptosomes and also involved in tissue homeostasis, aging, various pathological processes and irreversible cell injury $(4,5)$. The regulation of apoptosis occurs through extrinsic and intrinsic pathways the extrinsic pathway is engaged through so-called death receptors at the cell surface (6-8). A misregulation or failure of apoptosis can result in the development of cancer and the resistance to antitumor treatment might be due to insensitivity to the induction of apoptosis. Therefore, apoptosis is a mechanism that needs to be exploited when developing new chemotherapies for cancer.

Carbonyl cyanide $m$-chlorophenylhydrazone (CCCP) is a protonophore that renders the mitochondrial inner membrane permeable for protons and causes dissipation of the proton gradient across the inner mitochondrial membrane. CCCP also causes uncoupling of mitochondria so that the transfer of electrons through the electron transfer chain is no longer linked to the adenosine triphosphate (ATP) production, due to the loss of the electrochemical gradient. CCCP-induced apoptosis has been reported in many cell lines such as Jurkatneo, FL5.12, HL-60, and ST486 (9-12). However, some studies have reported that CCCP alone does not induce apoptosis (13-15).

TRAIL is a recently identified member of the TNF family that also activates the cognate receptor molecules through trimerization in a similar manner to other TNF family members $(16,17)$. DR4/TRAIL-R1 and DR5/TRAIL-R2 are intact TRAIL-Rs through which various apoptotic signals are transmitted into the cytoplasm. In contrast, DcR1 and DcR2 are truncated TRAIL-Rs with a deletion of the cytoplasmic regions containing the death domains. TRAIL has a unique selectivity for triggering apoptosis in tumor cells by engaging apoptosis via recruitment and rapid activation of caspase- 8 and might be less active against normal cells. However, the role of ROS 
in TRAIL-induced apoptosis is yet to be clearly defined. Recent preclinical studies have demonstrated that the repeated systemic administration of the recombinant TRAIL protein effectively limited tumor growth without any serious side effects $(18,19)$. As a result, TRAIL has attracted considerable attention as a promising treatment for human cancer.

Mitochondria play an important role in the intrinsic pathway of apoptosis. A number of changes occur in mitochondria following apoptotic signals such as loss of mitochondrial transmembrane potential (MTP, $\Delta \Psi \mathrm{m})$, generation of reactive oxygen species (ROS), opening of the permeability transition (PT) pore and release of apoptotic factors into the cytosol $(20,21)$. ROS are known to play a dual role in biological systems, since they can be either harmful or beneficial to living systems. Present study investigated the effect of mitochondrial uncoupling on TRAIL-induced apoptosis in TRAIL-resistant MCF-7 cell line.

\section{Materials and methods}

Cell culture and reagents. MCF-7 (human breast adenocarcinoma) cells were obtained from the ATCC (The Global Bioresource Center) and were maintained in RPMI-1640 (Gibco BRL, USA) culture medium supplemented with $10 \%$ (v/v) fetal bovine serum and antibiotics $(100 \mu \mathrm{g} / \mathrm{ml}$ penicillinstreptomycin). The cell cultures were incubated in an atmosphere containing $5 \% \mathrm{CO}_{2}$ at $37^{\circ} \mathrm{C}$. Carbonyl cyanide $m$-chlorophenylhydrazone (CCCP, Sigma) was dissolved in dimethylsulfoxide (DMSO) at a concentration of $20 \mathrm{mM}$ and was used at a final concentration of 5-80 $\mu \mathrm{M}$. N-acetyl-Lcysteine (NAC) was purchased from Sigma Chemicals Co. (St. Louis, MO, USA). 2',7'-Dichlorofluorescin diacetate and 5,5',6,6'-tetrachlo-1,1'3,3'-tetraethylbenzimidazol-carbocyanine iodide (JC-1) were purchased from Molecular Probes (Eugene, OR, USA).

Cell viability test. $\mathrm{MCF}-7$ cells were plated at $1.0 \times 10^{4}$ cells in a 12 -well plate, and incubated at $37^{\circ} \mathrm{C}$ for $24 \mathrm{~h}$. The cells were pretreated with different doses $(0,5,10,20,40$ and $80 \mu \mathrm{M})$ of carbonyl cyanide $m$-chlorophenylhydrazone (CCCP, Sigma) for $6 \mathrm{~h}$ and subsequently treated with TRAIL protein for $3 \mathrm{~h}$. Cells were also pretreated with an anti-oxidant NAC $(1 \mathrm{mM})$ for $1 \mathrm{~h}$ then followed by CCCP $(20 \mu \mathrm{M})$ for $6 \mathrm{~h}$ and TRAIL for additional $3 \mathrm{~h}$. The cell morphology was photographed under an optical microscope, and the cell viability was determined using the crystal violet staining method as described elsewhere (22). Briefly, the cells were stained with a staining solution $(0.5 \%$ crystal violet in $30 \%$ ethanol and $3 \%$ formaldehyde) for $10 \mathrm{~min}$ at room temperature, washed four times with water, and dried. Then the cells were lysed with a $1 \%$ SDS solution, and measured at $550 \mathrm{~nm}$. The cell viability was calculated from the relative dye intensity and compared with the controls.

Evaluation of mitochondrial transmembrane potential (MTP). The level of mitochondrial energization was determined using a lipophilic cation, 5,5',6,6'-tetrachlo-1,1'3,3'-tetraethylbenzimidazol-carbocyanine iodide (JC-1, Molecular Probes). This method is based on the ability of the fluorescent probe to enter the mitochondria selectively and reversibly change its color from green to orange as the mitochondrial potential increases. JC-1 exists as a monomer at low MTP values and shows a green fluorescence, while it forms an aggregate at a high MTP and shows a red fluorescence. Briefly, the cells were treated with NAC, CCCP and TRAIL for the indicated times, the cells were collected by centri-fugation, washed twice with a PBS buffer and resuspended in $500 \mu 1$ PBS containing JC-1 at a concentration of $10 \mu \mathrm{M}$. After $30 \mathrm{~min}$ incubation at $37^{\circ} \mathrm{C}$, the cells were photographed using a fluoroscope and the red fluorescence was monitored with a fluorescence plate reader (SpectraMax fluorometer with SoftMax Programme, Molecular Probes, USA) at 490 and $590 \mathrm{~nm}$ as excitation and emission wavelengths, respectively.

Determination of reactive oxygen species. 2',7'-Dichlorofluorescin diacetate (DCFH-DA), a non-fluorescent probe that turns to highly fluorescent 2 ', 7 '-dichlorofluorescin on reaction with ROS was used to monitor the production of ROS. Briefly, after the treatment, media were collected in a 96-well microplate and diluted DCFH-DA at a concentration of $10 \mu \mathrm{M}$ was added to each well. After $30 \mathrm{~min}$ incubation at $37^{\circ} \mathrm{C}$, DCFH fluorescence was determined with a fluorescence plate reader (SpectraMax fluorometer with SoftMax Programme, Molecular Probes, USA) at 490 and $525 \mathrm{~nm}$ as excitation and emission wavelengths, respectively.

Western blot assay. The whole cell lysates were prepared by harvesting the MCF-7 cells, washing them in cold phosphatebuffered saline, resuspending them in a lysis buffer $[25 \mathrm{mM}$ HEPES (pH 7.4), $100 \mathrm{mM}$ EDTA, $5 \mathrm{mM} \mathrm{MgCl}, 0.1 \mathrm{mM}$ DTT, and protease inhibitor mixture] and sonicating the lysate. The proteins $(30 \mu \mathrm{g})$ were separated on a $12-15 \%$ SDS gel and transferred to a nitrocellulose membrane. After incubation for $1 \mathrm{~h}$ with a 1:1000 primary antibody dilution buffer $(1 \%$ milk with PBS-Tween), the membranes were developed by enhanced chemiluminescence using a secondary antibody dilution. Bax protein was probed using the antibody obtained from BD Pharmingen (San Diego, CA).

Cellular fractionation. MCF-7 cells were resuspended in mitochondrial buffer (210 mM sucrose, $70 \mathrm{mM}$ mannitol, $1 \mathrm{mM}$ EDTA, $10 \mathrm{mM}$ HEPES), broken by six passages through a 25-guage needle and subjected to centrifugation at $700 \mathrm{~g}$ to pellet nuclei. The post nuclear supernatant was centrifuged at $10000 \mathrm{~g}$ for $30 \mathrm{~min}$ to pellet the mitochondrial enriched heavy membrane fraction and the supernatant was used as cytosolic fraction. Total proteins were subjected to Western blot analysis.

\section{Results}

MCF-7 cells are sensitized to TRAIL-induced apoptosis by uncoupler of oxidative phosphorylation in dose-dependent manner. To investigate the effects of uncoupling mitochondrial respiration on tumor cell response to TRAIL, we analysed the effects of CCCP, as a classic uncoupler of oxidative phosphorylation on TRAIL-induced apoptosis in MCF-7 cells. The cells were pretreated with indicated doses of CCCP for $6 \mathrm{~h}$, and then treated with TRAIL protein for additional 3 h. As shown in Fig. 1, CCCP enhanced TRAIL-induced 
A

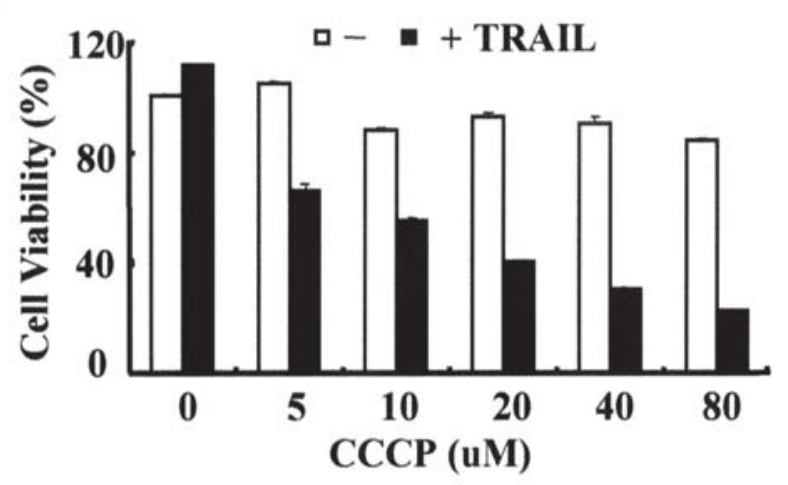

B

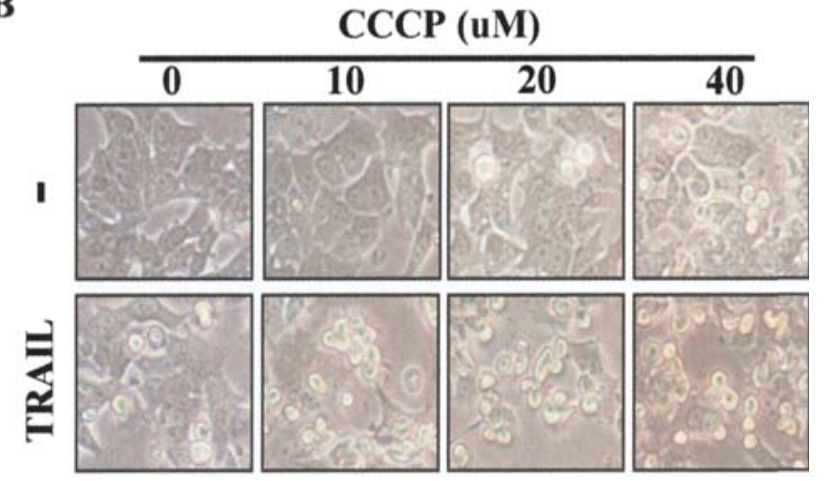

Figure 1. CCCP pretreatment enhances TRAIL-induced apoptotic cell death in a dose-dependent manner. (A) MCF-7 cells plated in 12 wells were treated with CCCP for the indicated doses for $6 \mathrm{~h}$ and then with TRAIL (300 ng) for an additional $3 \mathrm{~h}$. Cell viability was determined by crystal violet staining method. Viability of control cells was set at $100 \%$, and viability relative to the control was presented. The experiments were performed in triplicated at least twice. (B) MCF-7 cells were treated as described in (A). Cell morphology was photographed by a microscope (x200).

apoptosis in a dose-dependent manner. MCF-7 cells were found to be resistant to TRAIL protein but CCCP markedly enhanced TRAIL-induced apoptosis at different concentrations (Fig. 1A). The cell morphology also supported this enhanced effect of CCCP, showing that a CCCP pretreatment at all doses increased the number of apoptotic cells compared with that of CCCP and TRAIL alone (Fig. 1B). Thus, mitochondrial uncoupling sensitized TRAIL-resistant MCF-7 cells to TRAIL-induced apoptosis.

CCCP-induced ROS generation enhanced TRAIL-induced apoptosis in MCF-7 cells. To examine the role of ROS generation in TRAIL induced apoptosis, MCF-7 cells were treated with NAC before CCCP treatment and subsequently treated with TRAIL protein. Fig. 2A, clearly shows that NAC inhibited the enhanced effect of CCCP on TRAIL induced apoptosis. CCCP enhanced TRAIL effect and reduced the cell viability by $70 \%$ as compared to that of the negative control while NAC restored the cell viability to $70 \%$ (Fig. 2A). The generation of ROS was examined by using 2',7'-dichlorofluorescin fluorescence as described in Materials and methods. CCCP and CCCP with the TRAIL induced ROS generation compared to control and TRAIL alone (Fig. 2B). As expected, NAC pretreatment blocked the ROS generation in the cells pretreated with CCCP and then TRAIL and also prevented the enhanced
$\mathbf{A}$

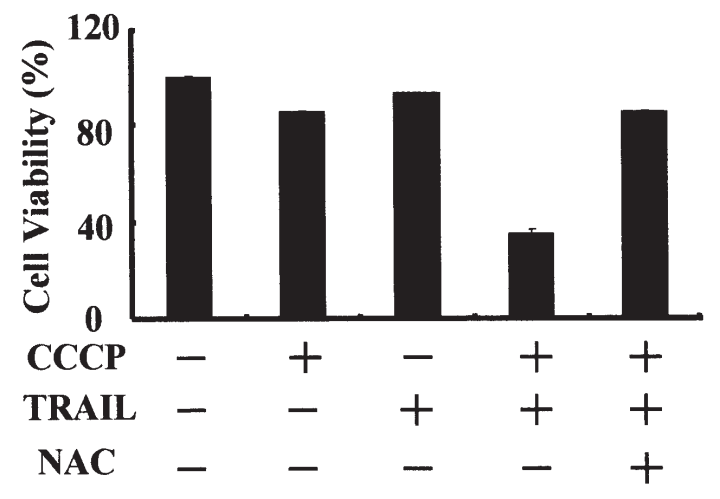

B

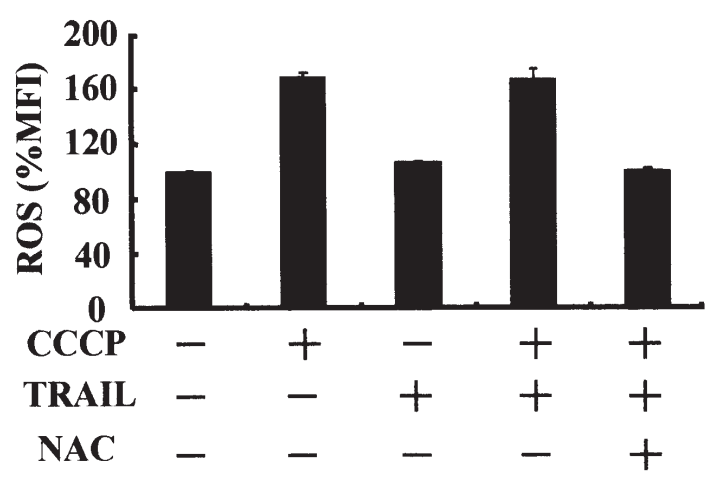

Figure 2. ROS generation regulate the enhanced effect of CCCP on TRAILinduced apoptosis. (A) MCF-7 cells plated in 12 wells were pretreated with CCCP $(20 \mu \mathrm{M} / \mathrm{ml})$ for $6 \mathrm{~h}$, and then coincubated with or without recombinant TRAIL protein $(300 \mathrm{ng} / \mathrm{ml})$ for an additional $2 \mathrm{~h}$. NAC $(1 \mathrm{mM})$ was added $1 \mathrm{~h}$ before CCCP treatment. Cell viability was determined by crystal violet staining method. Viability of control cells was set at $100 \%$, and viability relative to the control is presented. The experiments were performed at triplicate, at least twice. (B) MCF-7 cells were pretreated with CCCP $(20 \mu \mathrm{M} /$ $\mathrm{ml}$ ) for $2 \mathrm{~h}$, and then coincubated with or without recombinant TRAIL protein $(300 \mathrm{ng} / \mathrm{ml})$ for additional $2 \mathrm{~h}$. ROS generation was measured in media by using a fluorescent dye 2 ', 7 '-dichlorofluorescin diacetate $(10 \mu \mathrm{M})$. After incubation for $30 \mathrm{~min}$, ROS level was measured using a spectrophotometer.

effect of CCCP on TRAIL-induced apoptosis by restoring cell viability.

CCCP pretreatment induced loss in mitochondrial transmembrane potential $(\Delta \Psi \mathrm{m})$. We investigated the effect of CCCP on mitochondrial transmembrane potential. MCF-7 cells were pretreated with CCCP for $2 \mathrm{~h}$ followed by TRAIL protein for $2 \mathrm{~h}$. Photomicrographs indicate that CCCP and CCCP with TRAIL caused significant dissipation of $\triangle \Psi \mathrm{m}$ as compared with that of the negative control and TRAIL alone (Fig. 3). The fluoroscopic results presented in Fig. 3A shows the cells with green fluorescence after the CCCP and CCCP with TRAIL treatment indicating lower $\triangle \Psi \mathrm{m}$. In contrast, the negative control cells and the cells treated with TRAIL alone showed a red fluorescence, indicating high MTP values. Thus, MCF-7 cells did not show reduction in $\Delta \Psi \mathrm{m}$ after TRAIL treatment but combined treatment of CCCP and TRAIL significantly reduced $\Delta \Psi \mathrm{m}$. Therefore we investigated further the effect of NAC treatment on CCCP-induced loss in $\triangle \Psi \mathrm{m}$. As shown in Fig. 3A, NAC treatment restored the loss of $\Delta \Psi \mathrm{m}$ by CCCP and TRAIL treatment. NAC also inhibited the 
A
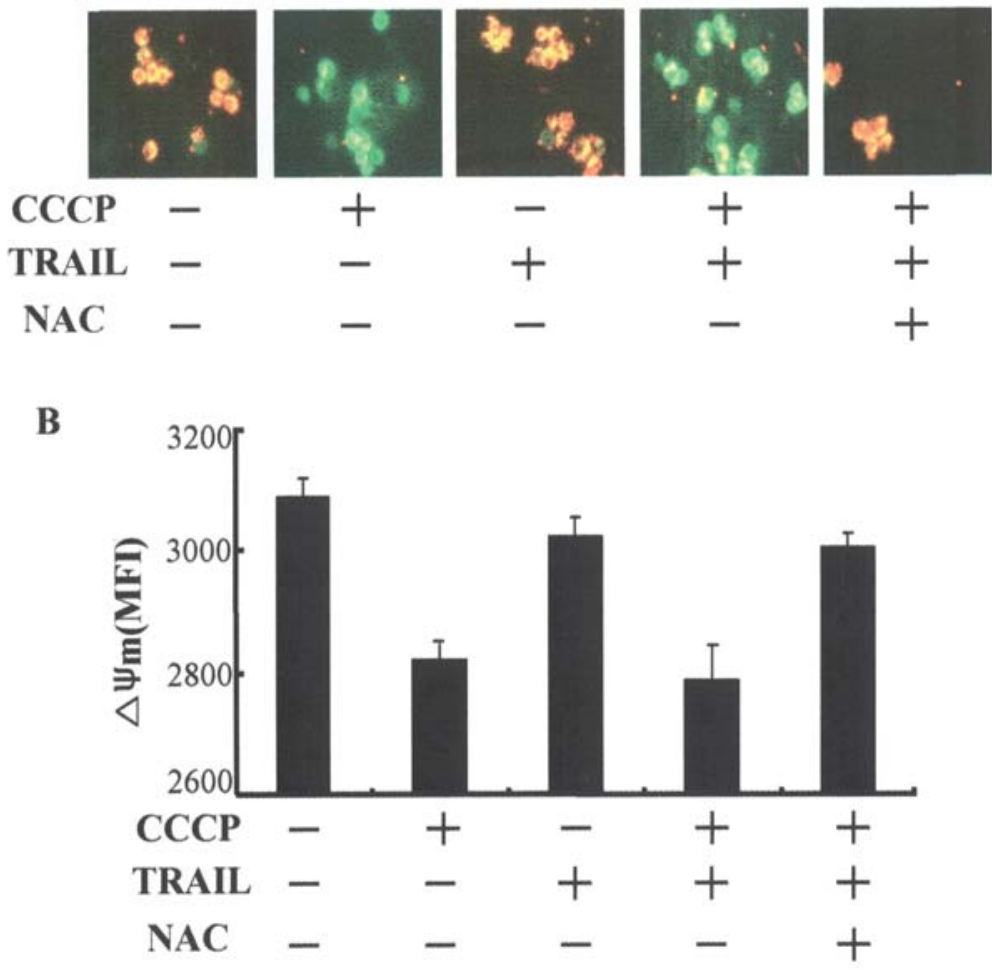

Figure 3. The change in mitochondrial transmembrane potential $(\Delta \Psi \mathrm{m})$ after pretreatment of CCCP with TRAIL. (A) MCF-7 cells plated in 6 wells were pretreated with CCCP $(20 \mu \mathrm{M} / \mathrm{ml})$ for $2 \mathrm{~h}$, and then coincubated with or without recombinant TRAIL protein (300 ng/ml) for additional $2 \mathrm{~h}$. MTP was determined using a JC-1 probe. The cells were photographed using a fluoroscope. (B) The red fluorescence intensity was measured 590 nm (emission of JC-1 aggregate) when excited at $490 \mathrm{~nm}$. For the each case, the mean fluorescence intensity values are indicated.

A

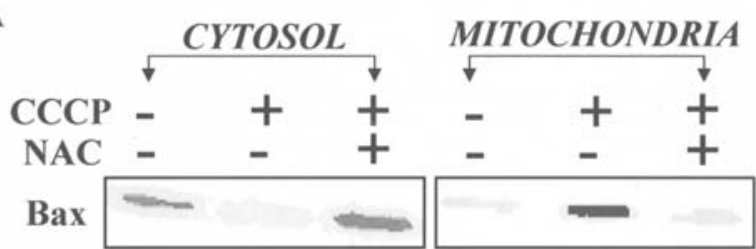

B

$\begin{array}{llllll}\text { CCCP } & - & + & - & + & + \\ \text { TRAIL } & - & - & + & + & + \\ \text { NAC } & - & - & - & - & + \\ \text { Bax } & & & & & \end{array}$

Figure 4. CCCP pretreatment leads to Bax translocation and enhances Bax protein expression. (A) MCF-7 cells were pretreated with CCCP $(20 \mu \mathrm{M} /$ $\mathrm{ml})$ for $2 \mathrm{~h}$ and NAC $(1 \mathrm{mM})$ was added $1 \mathrm{~h}$ before CCCP treatment. Cell lysates for mitochondria and cytosol were prepared, protein sample $(40 \mu \mathrm{g})$ was separated on SDS gel, analyzed for Bax protein translocation by Western blot analysis. (B) After CCCP $(20 \mu \mathrm{M} / \mathrm{ml})$ pretreatment for $2 \mathrm{~h}$, MCF-7 cells were coincubated with or without recombinant TRAIL protein $(300 \mathrm{ng} / \mathrm{ml})$ for an additional $2 \mathrm{~h}$. NAC $(1 \mathrm{mM})$ was added $1 \mathrm{~h}$ before CCCP treatment. Whole cell lysates were prepared and protein samples $(40 \mu \mathrm{g})$ were separated on SDS gel, analyzed for Bax protein by Western blot analysis.

dissipation of $\Delta \Psi \mathrm{m}$ induced by CCCP alone (data not shown) The fluorescence values clearly indicate that the cells treated with CCCP and CCCP with TRAIL showed low red fluorescence as compared with TRAIL and negative control (Fig. 3B). However, treatment with NAC followed by CCCP with TRAIL showed higher red fluorescence values than that of CCCP with TRAIL treatment. Overall, combine treatment of CCCP and TRAIL reduced $\triangle \Psi \mathrm{m}$ in MCF-7 cells, which was restored after NAC treatment.

Anti-oxidant $N$-acetyl-L-cysteine (NAC) prevents Bax translocation. MCF-7 cells were found to be resistant to TRAILinduced apoptosis even at high concentration (300 ng) but CCCP significantly enhanced TRAIL activity at different doses. Therefore, in order to investigate the apoptotic proteins involved, cytosolic and mitochondrial fractions were examined for Bax protein expression. After the treatment time with NAC, CCCP and TRAIL, cytosolic and mitochondrial fractions were prepared as indicated in Material and methods and subjected to Western blot analysis. As shown in Fig. 4A, $\mathrm{CCCP}$ translocated Bax protein from cytosol to mitochondria and the translocation of Bax protein was inhibited by NAC treatment. We also examined the expression of total Bax protein (Fig. 4B). The combined treatment of CCCP and TRAIL enhanced Bax protein expression and NAC also abrogated the activation of Bax protein thus corresponding with the results of ROS generation and $\Delta \Psi \mathrm{m}$.

\section{Discussion}

The role of dissipation of $\Delta \Psi \mathrm{m}$ and ROS generation in TRAILinduced apoptosis is yet to be clearly defined. In this study, we 
have shown that CCCP enhanced TRAIL-induced apoptosis in MCF-7 cells via dissipation of $\triangle \Psi \mathrm{m}$ and ROS generation. Moreover, CCCP treatment led to Bax translocation to mitochondria in MCF-7 cells and the translocation of Bax protein was inhibited by NAC treatment. CCCP specifically act to dissipate the proton gradient across the inner mitochondrial membrane thus known as mitochondrial poison. There are conflicting reports for apoptosis induced by CCCP alone in variety of cell lines. For example, CCCP alone did not induce apoptosis in human osteosarcoma cell lines (23) and SKW6 cells (15) while induced apoptosis in Jukat-neo, FL5.12 (9). In the present study, although CCCP alone reduced $\triangle \Psi \mathrm{m}$ and induced ROS generation it did not induce apoptosis of its own account.

Previous studies have reported that uncoupling the mitochondrial respiration with CCCP enhanced the effect of various chemotherapeutic drugs. Exposure of CCCP-treated human osteosarcoma cells to staurosporine or etoposide resulted in rapid cell death accompanied by cytochrome c release and Bax association (23). Moreover, CCCP was found to enhance the apoptosis-inducing ability of Fas/APO-1/CD95 signaling in Jurkat and CEM cells without causing apoptotic changes of its own account (15). But there are very few reports on the effect of CCCP on TRAIL-induced apoptosis. It has been suggested that uncoupling the mitochondrial respiration sensitize the leukemic cells to TRAIL-induced apoptosis (13). Also, human colon carcinoma cells were sensitized to TRAIL-induced apoptosis after CCCP pretreatment (14).

We investigated the effect of CCCP pretreatment on TRAIL-induced apoptosis in MCF-7 cells. CCCP alone and in combination with TRAIL caused the dissipation of $\Delta \Psi \mathrm{m}$ and ROS generation. In human colon carcinoma cells, the combined treatment of CCCP and TRAIL abrogated the ROS production by the complete loss of $\Delta \Psi \mathrm{m}$ (14). But here we found that CCCP treatment with TRAIL enhanced ROS generation by reducing $\triangle \Psi \mathrm{m}$ in MCF-7 cells.

$\mathrm{N}$-acetyl-1-cysteine (NAC) is an anti-oxidant drug and mainly known as a ROS scavenger. It reduces ROS generation and protects the cells from oxidative stress. Enhanced effect of CCCP on TRAIL-induced apoptosis was accompanied by dissipation of $\Delta \Psi \mathrm{m}$ and ROS generation. It has been reported that arsenic trioxide-induced loss of $\Delta \Psi \mathrm{m}$ was inhibited by NAC treatment (24) and in this study, CCCP induced loss in $\triangle \Psi \mathrm{m}$ and ROS generation that sensitized MCF-7 cells to TRAIL-induced apoptosis. We used NAC as an anti-oxidant to investigate the role of $\Delta \Psi \mathrm{m}$ and ROS generation. NAC treatment not only reduced ROS generation but also restored loss in $\Delta \Psi \mathrm{m}$. This study also found that NAC treatment restored the dissipation of $\triangle \Psi \mathrm{m}$ in MCF-7 cells when treated with CCCP and TRAIL together. All together, this indicates that besides scavenging the ROS, NAC also inhibited dissipation of $\Delta \Psi \mathrm{m}$ and thereby reduced enhanced effect of CCCP on TRAIL-induced apoptosis in MCF-7 cell line.

CCCP alone did not induce translocation of Bax protein in human osteosarcoma cells (23). Our results demonstrate that CCCP treatment translocated Bax protein from cytosol to mitochondria in MCF-7 cells. There are different reports for CCCP-induced apoptosis in a variety of cell lines (9-12). The present study showed that CCCP did not induce apoptosis of its own account because of expression of antiapoptotic proteins such as Bcl-2 and IAP-2 (data not shown). Collectively, our data suggest that CCCP pretreatment enhanced TRAIL effect by lowering $\triangle \Psi \mathrm{m}$ and ROS generation and thereby translocates Bax protein in MCF-7 cells.

In conclusion, ROS generation enhanced TRAIL-induced apoptosis in MCF-7 cells after CCCP treatment via Bax translocation. In addition, CCCP-induced ROS generation regulates the loss in MTP and Bax translocation. Thus, the combined treatment of CCCP and TRAIL might be useful in cancer therapy, particularly in TRAIL resistant cells. And therefore CCCP can be considered as a successful chemotherapeutic agent for ligand tumor therapy using TRAIL.

\section{Acknowledgements}

This study was supported by the Regional Research Centers Program of the Korean Ministry of Education and Human Resources Development through the Center for Healthcare Technology Development and 2nd stage Brain Korea 21 project and Korea Science and Engineering Foundation grant (NO. R01-2004-000-10459-0).

\section{References}

1. Ellis RE, Yuan JY and Horvitz HR: Mechanisms and functions of cell death. Annu Rev Cell Biol 7: 663-698, 1991.

2. Raff MC: Social controls on cell survival and cell death. Nature 356: 397-400, 1992.

3. Wyllie AH, Kerr JF and Currie AR: Cell death: the significance of apoptosis. Int Rev Cytol 68: 251-306, 1980.

4. Jacobson MD, Weil M and Raff MC: Programmed cell death in animal development. Cell 88: 347-354, 1997.

5. Raff MC: Size control: the regulation of cell numbers in animal development. Cell 86: 173-175, 1996.

6. Carson DA and Ribeiro JM: Apoptosis and disease. Lancet 341: 1251-1254, 1993

7. Carson DA and Tan EM: Apoptosis in rheumatic disease. Bull Rheum Dis 441: 1-3, 1995.

8. Nagata S: Apoptosis by death factor. Cell 88: 355-365, 1997.

9. De Graaf AO, van den Heuvel LP, Dijkman HB, De Abreu RA, Birkenkamp KU, De Witte T, van der Reijden BA, Smeitink JA and Jansen JH: Bcl-2 prevents loss of mitochondria in CCCPinduced apoptosis. Exp Cell Res 299: 533-540, 2004.

10. Mlejnek P: Caspase- 3 activity and carbonyl cyanide mchlorophenylhydrazone-induced apoptosis in HL-60. Altern Lab Anim 29: 243-249, 2001.

11. Muscarella DE, O'Brien KA, Lemley AT and Bloom SE: Reversal of Bcl-2-mediated resistance of the EW36 human Bcell lymphoma cell line to arsenite- and pesticide-induced apoptosis by PK11195, a ligand of the mitochondrial benzodiazepine receptor. Toxicol Sci 74: 66-73, 2003.

12. O'Brien KA, Muscarella DE and Bloom SE: Differential induction of apoptosis and MAP kinase signaling by mitochondrial toxicants in drug-sensitive compared to drug-resistant B-lineage lymphoid cell lines. Toxicol Appl Pharmacol 174: 245-256, 2001.

13. Hao JH, Yu M, Liu FT, Newland AC and Jia L: Bcl-2 inhibitors sensitize tumor necrosis factor-related apoptosis-inducing ligandinduced apoptosis by uncoupling of mitochondrial respiration in human leukemic CEM cells. Cancer Res 64: 3607-3616, 2004.

14. Izeradjene K, Douglas L, Tillman DM, Delaney AB and Houghton JA: Reactive oxygen species regulate caspase activation in tumor necrosis factor-related apoptosis-inducing ligandresistant human colon carcinoma cell lines. Cancer Res 65: 7436-7445, 2005.

15. Linsinger G, Wilhelm S, Wagner H and Hacker G: Uncouplers of oxidative phosphorylation can enhance a Fas death signal. Mol Cell Biol 19: 3299-3311, 1999.

16. Wiley SR, Schooley K, Smolak PJ, et al: Identification and characterization of a new member of the TNF family that induces apoptosis. Immunity 3: 673-682, 1995. 
17. Pitti RM, Marsters SA, Ruppert S, Donahue CJ, Moore A and Ashkenazi A: Induction of apoptosis by Apo-2 ligand, a new member of the tumor necrosis factor cytokine family. J Biol Chem 271: 12687-12690, 1996.

18. Walczak H, Miller RE, Ariail K, Gliniak B, Griffith TS, Kubin M, Chin W, Jones J, Woodward A, Le T, Smith C, Smolak P, Goodwin RG, Rauch CT, Schuh JC and Lynch DH: Tumoricidal activity of tumor necrosis factor-related apoptosisinducing ligand in vivo. Nat Med 5: 157-163, 1999.

19. Griffith TS, Anderson RD, Davidson BL, Williams RD and Ratliff TL: Adenoviral-mediated transfer of the TNF-related apoptosis-inducing ligand/Apo-2 ligand gene induces tumor cell apoptosis. J Immunol 165: 2886-2894, 2000.

20. Kroemer G and Reed JC: Mitochondrial control of cell death. Nat Med 6: 513-519, 2000.

21. Newmeyer DD and Ferguson-Miller S: Mitochondria: releasing power for life and unleashing the machineries of death. Cell 112: 481-490, 2003
22. Park SY, Billiar TR and Seol DW: Hypoxia inhibition of apoptosis induced by tumor necrosis factor-related apoptosisinducing ligand (TRAIL). Biochem Biophys Res Commun 291: $150-153,2002$.

23. Lim ML, Minamikawa T and Nagley P: The protonophore CCCP induces mitochondrial permeability transition without cytochrome c release in human osteosarcoma cells. FEBS Lett 503: 69-74, 2001.

24. Kang YH, Yi MJ, Kim MJ, Park MT, Bae S, Kang CM, Cho CK, Park IC, Park MJ, Rhee CH, Hong SI, Chung HY, Lee YS and Lee SJ: Caspase-independent cell death by arsenic trioxide in human cervical cancer cells: reactive oxygen species-mediated poly(ADP-ribose) polymerase-1 activation signals apoptosisinducing factor release from mitochondria. Cancer Res 64: 8960-8967, 2004. 